CERN-PH-TH/2012-113

\title{
String in AdS Black Hole: A Thermo Field Dynamic Approach
}

\author{
M. Botta Cantcheft* \\ IFLP-CONICET CC 67, 1900, La Plata, Buenos Aires, Argentina and \\ CERN, Theory Division, 1211 Geneva 23, Switzerland \\ Alexandre L. Gadelhat \\ Instituto de Física, Universidade Federal da Bahia, \\ Campus Universitário de Ondina, CEP: 40210-340, Salvador, BA, Brasil \\ Dáfni F. Z. Marchiord $\sharp$ and Daniel Luiz Nede \\ Universidade Federal do Pampa, Campus Bagé, Travessa 45, \\ número 1650 - Bairro Malafaia, CEP: 96413-170, Bagé, RS, Brasil
}

\begin{abstract}
Based on Maldacena's description of an eternal AdS-black hole, we reassess the Thermo Field Dynamics (TFD) formalism in the context of the AdS/CFT correspondence. The model studied here involves the maximally extended AdS-Schwarschild solution and two (non-interacting) copies of the CFT associated to the global AdS spacetime, along with an extension of the string by imposing natural gluing conditions in the horizon. We show that the gluing conditions in the horizon define a string boundary state which is identified with the TFD thermal vacuum, globally defined in the Kruskal extension of the AdS black hole. We emphasize the connection of this picture with unitary SU(1,1) TFD formulation and we show that information about the bulk and the conformal boundary is present in the $\mathrm{SU}(1,1)$ parameters. Using the unitary $\mathrm{SU}(1,1)$ TFD formulation, a canonical prescription for calculating the worldsheet real time thermal Green's function is made and the entropy associated with the entanglement of the two CFT's is calculated.
\end{abstract}

\footnotetext{
*bottac@cern.ch, botta@fisica.unlp.edu.ar

$\dagger$ agadelha@ufba.br

$\ddagger$ dafnimarchioro@unipampa.edu.br

$\S$ daniel.nedel@unipampa.edu.br
} 


\section{INTRODUCTION}

Since its advent, the holographic AdS/CFT correspondence has been exploited to study the physics of non-Abelian quark-gluon plasmas at finite temperature from bulk gravitational physics [1 4]. More recently, it has been emphasized that one can also exploit the gravitational description to understand the hydrodynamic regime of the quark-gluon plasma and the quark Brownian motion in a conformal fluid [5]. In these applications, the string propagates in an asymptotically AdS space containing black holes, and the quark physics is described by an open string stretching from the horizon up to a probe brane placed to a short distance (related to the ultraviolet cut-off) from the conformal boundary. The quark is naturally identified with the end of the string on the boundary, while near the Black Hole horizon it is supposed to end on an effective membrane with thermal and dissipative properties, called stretched horizon. In this scenario, the Hawking radiation induces random motion on the string end point. Then, the motion of the quark in the quark-gluon plasma is assumed to be described by a Langevin equation, whose parameters can then be deducted by bulk calculations. In principle, such investigations would require an exact first quantization of the string in an AdS black hole, which in general is a very difficult task. In spite of this problem, much progress has been made by studying small perturbations of the Nambu-Goto action up to quadratic order 1 This approximation corresponds to the non relativistic limit and, in this context, the relativistic dynamics of the quark-gluon plasma at finite temperature cannot be described.

In general, since the bulk geometry has an event horizon, the induced metric on the string worldsheet also corresponds to a black hole geometry [8] and, owing to Hawking radiation, the problem of studying small fluctuations of the Nambu-Goto action in AdS black holes reduces to the dynamics of two dimensional quantum field at finite temperature. So, in order to understand completely how the AdS/CFT at finite temperature works, it is necessary to understand this thermal field theory on the worldsheet. In [9] a prescription was formulated for computing the real-time Green's functions at finite temperature. This was realized in the context of the Schwinger-Keldysh formalism, by using the Kruskal extension of the AdS Schwarzschild spacetime. In addition to Schwinger-Keldysh formalism, there is another real time formalism appropriated to explore the present context; it is the Thermo Field

\footnotetext{
${ }^{1}$ See [6], 7] and references therein.
} 
Dynamics (TFD) formalism [10]. TFD provides a picture where the degrees of freedom behind the horizon play an important role, which should be applied to describe strings in AdS black holes. This point of view was noticed by Israel [11], and put forth by Maldacena in the holographic context [12]. This is the picture that we intend to study here.

TFD is a real time formalism conceived originally to deal with thermal systems approaching them directly on their Fock space. The Takahashi and Umezawa original proposal consists of a canonical quantum field theory that reproduces the statistical averages of any system's observables [10]. In fact, from the statistical mechanics point of view, considering an observable operator $Q$, its statistical average is defined by the functional $\omega(Q)=\operatorname{Tr}(\rho Q)$ as

$$
\langle Q\rangle=\frac{\omega(Q)}{\omega(1)}=\frac{\operatorname{Tr}[Q \rho]}{\operatorname{Tr}[\rho]}
$$

where $\rho$ is the density operator of the system. Such functional is recognized as a state in the algebraic statistical mechanics with the operators obeying a $C^{*}$ algebra. In this point of view, the algebra equipped with a functional admits a reducible representation of the Hilbert space such as a Fock space [13-20] and the statistical average in TFD is written as

$$
\langle Q\rangle=\frac{\omega(Q)}{\omega(1)}=\langle 0(\beta)|Q| 0(\beta)\rangle
$$

with $|0(\beta)\rangle$ being the TFD thermal vacuum. More generally, the central idea is to construct a quantum field theory whose vacuum contains the information about the environment under which the system is subjected. Once observed that temperature is introduced as an external parameter, it is verified that the thermal vacuum appears as a boundary state in the doubled Fock space composed by the physical space of the system and a copy of it. The expression defining such a state is called thermal state condition [21] and it must contain all the information about the system to be considered. Starting from a physical system at zero temperature, TFD's general procedure consists of the doubling of degrees of freedom of the system and a suitable Bogoliubov transformation to entangle such duplicated degrees of freedom. With the doubling, one obtains an enlarged Hilbert space composed by the original and an auxiliary space, which is identical to the original one and related to the so called tilde system. The enlarged Hilbert space is denoted by a hat and is given by $\hat{\mathcal{H}}=\mathcal{H} \otimes \tilde{\mathcal{H}}$. The original and tilde systems are related by a mapping called tilde conjugation rules associated to the application of the Tomita-Takesaki modular operator of the statistical mechanics algebraic approach [15, 16]. 
The Bogoliubov transformation is obtained using a generator in such a way that, in a finite volume limit, the transformation is unitary and preserves the tilde conjugation rules. The thermal effects arise from the vacuum correlation introduced by the transformation over the enlarged system vacuum. This construction was the first one for TFD; however, one can find a set of generators that maintains the thermal nature of the transformation. The set of generators constructed to this end is shown to be a linear combination of operators that forms an oscillator representation of $\mathrm{SU}(1,1)$ group for bosons and $\mathrm{SU}(2)$ for fermions [21, 22]. These sets for fermionic and bosonic systems can be combined in at least two different ways, providing generalizations of the TFD approach. In one case the tilde conjugation rules are preserved but the transformation, even in a finite volume limit, is non-unitary. This construction was largely developed and its connection with other thermal field theories is clear as one can see, for example, in Refs. [21] and [24]. In the second case the transformation is unitary in a finite volume limit, but the tilde conjugation rules are not preserved. This is the so called general unitary TFD formulation, and it is still under investigation.

Effectively, the general unitary formulation was applied to a physical system in [25], where it was perceived that a systematic study about the formulation's implications was necessary. In references [26 29] such analysis are carried out and the formulation was successfully applied to describe superstrings at finite temperature2. Recently the general unitary formulation was also applied in Refs [59, 60], but considerations about interacting systems, dissipation, possible connection with other real time formalisms and with quantum statistical mechanics' algebraic approach appear as open questions. Here we go forward towards a better understanding of these constructions.

In this work we show that the $\mathrm{SU}(1,1)$ TFD formulation arises naturally for strings propagating in an AdS black hole geometry. This is achieved noting that, by gluing the string defined on the left quadrant of the Kruskal diagram (with the string defined on the right one), we get a string boundary state, which is exactly the thermal vacuum of TFD. This is in fact an entangled state and in the context of AdS/CFT, it is an entanglement of the two CFTs defined by the Kruskal extension of the AdS black hole. We explore the general unitary $\mathrm{SU}(1,1)$ formulation of TFD to show that all information of the system is present in the Bogoliubov parameters. Also the TFD entropy operator is used to obtain the

${ }^{2}$ It is important to draw attention that TFD was also applied to string theory in many others contexts in addition to those already mentioned [30-58]. 
entropy of the system, associated here to the presence of the horizon. Its high temperature limit is shown to be in agreement with the Cardy formula.

Keeping in mind this whole framework and the motivations mentioned above, we organize the present paper according to the following outline: in Section II, we present the model (described originally in [5]) and implement the Kruskal extension. In section III, in the spirit

of reference [6] we present the suitable generalization of the analytic continuation required to circumvent the conical singularity, and then define a string in all spacetime by gluing up the strings of the left and right quadrant. It is shown that the boundary state resulting from this procedure is an entangled state of elements of the two CFTs. In Section IV, the general unitary $\mathrm{SU}(1,1)$ TFD formulation is presented, and it is shown that the obtained vacuum state matches the boundary state coming from the gluing, which has the information about the conditions imposed over the string defined in all spacetime. The entropy is calculated and it is interpreted as a result of the entanglement of the CFTs; also, the thermal two-point functions are obtained. Finally, our final considerations and perspectives are presented in section $\mathrm{V}$.

\section{OPEN STRING IN THE ADS BLACK HOLE}

In this section we describe the basic features of the scenario that we are studying; a detailed discussion can be found in ref [5]. The model consists of putting a probe fundamental string stretched from the boundary to the horizon of the following three dimensional asymptotically AdS black hole geometry:

$$
d s^{2}=-\frac{r^{2}-r_{H}^{2}}{\ell^{2}} d t^{2}+\frac{r^{2}}{\ell^{2}} d X^{2}+\frac{\ell^{2}}{r^{2}-r_{H}^{2}} d r^{2}
$$

which is in fact the metric for a non rotating BTZ black hole. Here $t$ and $X$ are boundary coordinates. In this coordinate system, the open string is suspended from the boundary at $r=\infty$ and straight down along the $r$ direction into the horizon at $r=r_{H}$. Although we are focusing in this simple three dimensional case, in [5] the results are generalized for $d$-dimensional spacetimes. The Hawking temperature is

$$
T \equiv \frac{1}{\beta}=\frac{r_{H}}{2 \pi \ell^{2}}
$$

where $\ell$ is the AdS radius. 
Let us now write the string sigma model. For general planar $d$-dimensional AdS black holes, the metric can always be written as follow

$$
d s^{2}=g_{\mu \nu} d x^{\mu} d x^{\nu}+G_{I J} d X^{I} d X^{J}
$$

where both $g_{\mu \nu}$ and $G_{I J}$ are independent of $X^{I}$ and $x^{\mu}=t, x$. In the gauge that we are going to use, the worldsheet coordinates are identified with the spacetime coordinates $t$ and $x$ and only the transversal modes $X^{I}=X^{I}(t, x)$ are dynamical. In this gauge, the Nambu-Goto action can be written as

$$
S_{N G}=-\frac{1}{2 \pi \alpha^{\prime}} \int \sqrt{\operatorname{det} \gamma_{\mu \nu}}
$$

where the induced metric is $\gamma_{\mu \nu}=G_{I J} \partial_{\mu} X^{I} \partial_{\nu} X^{J}$. By expanding the Nambu-Goto action, we get a power series of $\partial_{t} X$ and $\partial_{x} X$ which produces worldsheet interactions. As we are going to study only small fluctuations of the equilibrium value $X^{I}=0$, only quadratic terms in the action are considered. Since this approximation implies the regime $\left|\partial_{t} X^{I}\right|<<1$ of small velocities, we are in fact taking the non-relativistic limit. In the quadratic approximation, the Nambu-Goto action can be written as

$$
S_{N G} \approx-\frac{1}{4 \pi \alpha^{\prime}} \int \sqrt{g(x)} g^{\mu \nu}(x) G_{I J}(x) \partial_{\mu} X^{I} \partial_{\nu} X^{J}
$$

where $g(x)=\operatorname{det} g_{\mu \nu}$.

Following reference [5], one returns to $A d S_{3}$ case, where there is only one transversal variable $X$, and writes the metric in terms of the tortoise coordinate $r_{\star}$,

$$
\begin{array}{r}
d s^{2}=\frac{r^{2}-r_{H}^{2}}{\ell^{2}}\left(-d t^{2}+d r_{\star}^{2}\right)+\frac{r^{2}}{\ell^{2}} d X^{2}, \\
r_{\star} \equiv \frac{\ell^{2}}{2 r_{H}} \ln \left(\frac{r-r_{H}}{r+r_{H}}\right)=\frac{\beta}{4 \pi} \ln \left(\frac{r-r_{H}}{r+r_{H}}\right)
\end{array}
$$

In this coordinate system, we can write the equations of motion in the quadratic approximation used in (7) as

$$
\left[-\partial_{t}^{2}+\frac{r^{2}-r_{H}^{2}}{l^{4} r^{2}} \partial_{r}\left(r^{2}\left(r^{2}-r_{H}^{2}\right) \partial_{r}\right)\right] X(t, r)=0
$$

Defining dimensionless quantities

$$
\rho \equiv \frac{r}{r_{H}}, \quad \nu \equiv \frac{l^{2} \omega}{r_{H}}=\frac{\beta \omega}{2 \pi}
$$

the linearly independent solutions to (9) are given by

$$
X(t, r)=e^{-i \omega t} g_{\omega}(r)
$$


with

$$
g_{\omega}^{( \pm)}=\frac{1}{1 \pm i \nu} \frac{\rho \pm i \nu}{\rho}\left(\frac{\rho-1}{\rho+1}\right)^{ \pm i \nu / 2}=\frac{1}{1 \pm i \nu} \frac{\rho \pm i \nu}{\rho} e^{ \pm i \omega r_{\star}}
$$

Because the string extends from the horizon of the black hole to the boundary, a cutoff is needed to prevent UV divergences in the boundary. Also, we need to regularize IR divergences at the horizon.

The UV divergences are controlled by imposing a Neumann boundary condition $\partial_{r} X=0$ at the cut-off surface:

$$
\rho=\rho_{c} \gg 1 \quad \text { or } \quad r=r_{c} \equiv r_{H} \rho_{c}
$$

Solving for $\left.\partial_{\rho} f_{\omega}\right|_{\rho=\rho_{c}}=0$, we find the value of $B$ in (19)):

$$
B=\frac{1-i \nu}{1+i \nu} \frac{1+i \rho_{c} \nu}{1-i \rho_{c} \nu}\left(\frac{\rho_{c}-1}{\rho_{c}+1}\right)^{i \nu} \equiv e^{i \theta_{\omega}}
$$

which is a pure phase. Next it is necessary to regulate the IR divergence at horizon $(\rho=1)$. This is achieved by putting an IR cut-off at $\rho_{s}=1+2 \epsilon$, with $\epsilon<<1$. As argued in [5], the effect of this regulator is to discretize the continuum spectrum which naturally occurs when considering horizon dynamics.

After regularizing the theory, we can find a normalized basis of modes and start quantizing $X(t, r)$ by expanding it in those modes:

$$
X(t, r)=\sum_{\omega>0}\left[a_{\omega} u_{\omega}(t, \rho)+a_{\omega}^{\dagger}\left[u_{\omega}(t, \rho)\right]^{*}\right]
$$

where

$$
u_{\omega}(t, \rho)=\sqrt{\frac{\alpha^{\prime} \beta}{2 \ell^{2} \omega \ln (1 / \epsilon)}}\left[g_{\omega}^{(+)}(\rho)+e^{i \theta_{\omega}} g_{\omega}^{(-)}(\rho)\right] e^{-i \omega t}
$$

and the coefficients of the expansion $a_{\omega}$ satisfy the relations:

$$
\left[a_{\omega}, a_{\omega^{\prime}}\right]=\left[a_{\omega}^{\dagger}, a_{\omega^{\prime}}^{\dagger}\right]=0 \quad\left[a_{\omega}, a_{\omega^{\prime}}^{\dagger}\right]=\delta_{\omega \omega^{\prime}}
$$

Near the horizon $(\rho \sim 1)$, the metric becomes plane and the solution (12) behaves like

$$
g_{\omega}^{( \pm)} \sim e^{ \pm i \omega r_{\star}}
$$

So, near the horizon the solutions are written in terms of ingoing and outgoing plane waves:

$$
X_{R}(t, r)=\sqrt{\frac{\alpha^{\prime} \beta}{2 \ell^{2} \omega \ln (1 / \epsilon)}} \sum_{\omega>0}\left\{a_{\omega}\left[e^{-i \omega\left(t-r_{\star}\right)}+e^{i \theta_{\omega}} e^{-i \omega\left(t+r_{\star}\right)}\right]\right\} .
$$


Let us now further develop this model in order to show how the TFD structure rises naturally in this scenario. The solution (15) can be written in terms of Kruskal coordinates, where the structure of the full Penrose diagram for this AdS space becomes apparent. Outside the horizon, there are two causally disconnected spacetime geometries (both of which are asymptotically AdS), defining two CFTs. In the Kruskal plane, the solutions are uniquely determined by their boundary conditions at the two Minkowski boundaries, in the right $(R)$ and, respectively, left $(L)$ quadrants of the Kruskal diagram. Also, in order to define the string in all spacetime, it is necessary to perform an appropriate analytical continuation in the Kruskal variables $U$ and $V$. The Kruskal coordinates are defined in terms of $t$ and $r_{\star}$ by the transformation

$$
t=\ln \left(\frac{V}{-U}\right) \quad r_{\star}=\ln (V(-U))
$$

where $(U, V)$ are defined such that $(V>0, U<0)$ in the right quadrant and $(V<0, U>0)$ in the left quadrant.

Note that, as any function of $t$ and $r$ can be written in terms of $U$ and $V$, we don't need to write the metric in terms of $U$ and $V$ and solve again the equation of motion. We can solve the string equation of motion separately in the $R$ and $L$ quadrants and obtain one set of mode functions in each quadrant. So, if we define the solution (15) in the $R$ quadrant, we just need to write the respective solution in the $L$ quadrant defining it as a copy of the $R$ solution:

$$
X_{L}(\tilde{t}, r)=\sum_{\omega>0}\left\{\tilde{a}_{\omega} \tilde{u}_{\omega}(\tilde{t}, \rho)+\tilde{a}_{\omega}^{\dagger}\left[\tilde{u}_{\omega}(\tilde{t}, \rho)\right]^{*}\right\}
$$

where

$$
\tilde{u}_{\omega}(\tilde{t}, \rho)=\sqrt{\frac{\alpha^{\prime} \beta}{2 \ell^{2} \omega \ln (1 / \epsilon)}}\left[g_{\omega}^{(+)}(\rho)+e^{i \tilde{\theta}_{\omega}} g_{\omega}^{(-)}(\rho)\right] e^{i \omega \tilde{t}}
$$

In order to relate these solutions, we need to impose boundary conditions at the horizon $U=V=0$, where the transformation (20) is singular. This is the topic of the next section. By doing the Kruskal extension, we have defined two CFTs. Although classically these CFTs are causally disconnected, we are going to show in the next sections that states of a CFT defined in one quadrant play a particular role in the expectation values of observables defined in the other quadrant. 


\section{BUILDING UP THE EXTENDED STRING FROM GLUING}

The Kruskal extension defines two asymptotically AdS spaces and two causally disconnected CFTs. By defining the string in all spacetime, we can quantum connect these two CFTs. In particular, we are going to show now that the string vacuum is an entangled state of states of the two CFTs and it is the thermal vacuum of the TFD.

In order to define continuously the string in all spacetime by connecting the solution in the $L$ quadrant to that in the R quadrant, we need to avoid the singularity at $U=V=0$ by performing a well known analytic continuation. In terms of the old coordinates, this analyt-

ical continuation relates $\tilde{t}-t=i \frac{\beta}{2}$, where $\tilde{t}$ is the time coordinate of the $L$ quadrant of the spacetime. The resulting thermal field theory ultimately reproduces the contour correlation functions for a specific choice of the Schwinger-Keldysh contour. As pointed out in [6], this analytical continuation can be generalized in order to take into account deformations of the usual Schwinger-Keldysh contour. Inspired by that observation, but taking in mind the TFD approach, our proposal is considering $\tilde{t}-t=i \alpha \beta$, for $\alpha$ a complex parameter. As it will be clear in the next section, the unitarity of the $\mathrm{SU}(1,1)$ TFD formulation will imply in $\alpha+\alpha^{*}=1$. Also, this constraint ensures the Kubo-Martin-Schwinger (KMS) condition and therefore the usual time periodicity of the correlation functions.

Near the horizon $(\rho \sim 1)$, the solutions (15) and (21) simplify to

$$
\begin{array}{r}
X_{R}(t, r)=\sqrt{\frac{\alpha^{\prime} \beta}{2 \ell^{2} \omega \ln (1 / \epsilon)} \sum_{\omega>0}\left\{a_{\omega}\left[e^{-i \omega\left(t-r_{\star}\right)}+e^{i \theta_{\omega}} e^{-i \omega\left(t+r_{\star}\right)}\right]\right.} \\
\left.+a_{\omega}^{\dagger}\left[e^{i \omega\left(t-r_{\star}\right)}+e^{-i \theta_{\omega}} e^{i \omega\left(t+r_{\star}\right)}\right]\right\}, \\
X_{L}(t, r)=\sqrt{\frac{\alpha^{\prime} \beta}{2 \ell^{2} \omega \ln (1 / \epsilon)} \sum_{\omega>0}\left\{\tilde{a}_{\omega}\left[e^{i \omega\left(\tilde{t}-r_{\star}\right)}+e^{-i \tilde{\theta}_{\omega}} e^{i \omega\left(\tilde{t}+r_{\star}\right)}\right]\right.} \\
\left.+\tilde{a}_{\omega}^{\dagger}\left[e^{-i \omega\left(\tilde{t}-r_{\star}\right)}+e^{i \tilde{\theta}_{\omega}} e^{-i \omega\left(\tilde{t}+r_{\star}\right)}\right]\right\} .
\end{array}
$$

Note that owing to reverse clockwise direction of the L quadrant, while the $a$ operator annihilates outgoing modes at the horizon in $R$ quadrant, the $a$ operator annihilates ingoing modes in the L quadrant. We are going to impose gluing conditions on a state called $\left|B_{\text {hor }}\right\rangle$, which represents that the string in the $R$ quadrant is connected with its dual copy in the $L$ quadrant. These conditions are

$$
\left(\left.X_{L}(t, r)\right|_{\rho=1}-\left.X_{R}(t, r)\right|_{\rho=1}\right)\left|B_{h o r}\right\rangle=0
$$


We avoid the singularity at $\rho=1$ using Kruskal coordinates and performing the analytic continuation $\tilde{t}=t+i \alpha \beta$. Solving for the gluing conditions, we have

$$
\begin{aligned}
\left(\tilde{a}_{\omega} e^{-\omega \alpha \beta}-a_{\omega}^{\dagger}\right)\left|B_{h o r}\right\rangle & =0 \\
e^{-i \theta_{\omega}}\left(\tilde{a}_{\omega} e^{-\omega \alpha \beta}-a_{\omega}^{\dagger}\right)\left|B_{h o r}\right\rangle & =0 \\
\left(a_{\omega}-\tilde{a}_{\omega}^{\dagger} e^{\omega \alpha \beta}\right)\left|B_{h o r}\right\rangle & =0 \\
e^{i \theta_{\omega}}\left(a_{\omega}-\tilde{a}_{\omega}^{\dagger} e^{\omega \alpha \beta}\right)\left|B_{h o r}\right\rangle & =0
\end{aligned}
$$

The state $\left|B_{h o r}\right\rangle$, which satisfy the equations (27), is:

$$
\left|B_{\text {hor }}\right\rangle=N \exp \left[\sum_{\omega} e^{-\alpha \beta \omega} a_{\omega}^{\dagger} \tilde{a}_{\omega}^{\dagger}\right]|0, \tilde{0}\rangle
$$

where $N$ is a normalization factor.

The boundary sate $\left|B_{h o r}\right\rangle$ is a string entangled state, which entangles string states defined in the $\mathrm{L}$ and $\mathrm{R}$ quadrants. In the AdS/CFT context, this state entangles the two boundary CFTs, in particular the heavy quark states defined by the endpoint of the string at the two asymptotic boundaries.

Notice that these states are built up by imposing boundary conditions, referred here as gluing conditions. A similar construction in the Minkowski spacetime was done in Ref. [61]. It has been also argued that these states are equivalent to ordinary boundary states in the closed string Hilbert space under a worldsheet transformation of the one-loop diagram in Euclidean time [61].

In the next section we are going to show that $\left|B_{h o r}\right\rangle$ can also be interpreted as the thermal vacuum in the point of view of Thermo Field Dynamics (TFD), where the equilibrium temperature is the Hawking temperature. Expectation values of the $\mathrm{R}$ string states on this state correspond to statistical averages in an ensemble of open string states. In the context of the AdS/CFT conjecture, this imply that we can calculate non-perturbatively thermodynamical properties of the CFT through the calculation of the expectation values in this state. 


\section{THE BOGOLIUBOV TRANSFORMATION, ENTROPY OPERATOR AND THERMAL TWO POINT FUNCTION}

Let us show now that the boundary state defined in the previous section can be achieved from a Bogoliubov transformation and that this state is also defined by an entanglement entropy operator. Indeed, the TFD approach appears naturally in this scenario. As a matter of fact, the general unitary $S U(1,1)$ TFD formulation will be used in order to fit the generalization proposed for the analytic continuation.

The presence of the horizon defines two string Hilbert spaces, related to the two regions $L$ and $R$. In the following we will refer to the elements of these regions as tilde and non-tilde, respectively. The total Hilbert space is the tensor product of the two spaces $\mathcal{H}_{o} \otimes \widetilde{\mathcal{H}}_{o}$, where in this case $\mathcal{H}_{o}$ is the Hilbert space built with cyclic applications of the operators $a_{\omega}, a_{\omega}^{\dagger}$ while the $\widetilde{\mathcal{H}}_{o}$ Hilbert space is related to the $\tilde{a}_{\omega}, \tilde{a}_{\omega}^{\dagger}$ operators. The standard vacuum in this extended theory is defined by

$$
\left.\left.a_{\omega}|0\rangle\right\rangle=\tilde{a}_{\omega}|0\rangle\right\rangle=0
$$

with $|0\rangle\rangle=|0\rangle \otimes|\tilde{0}\rangle$ as usual.

Owing to the reverse clockwise direction of the L quadrant, the total worldsheet Hamiltonian is defined as $\widehat{H}=H-\widetilde{H}$, where the $H$ Hamiltonian is proportional to number operator $N_{\omega}=a_{\omega}^{\dagger} a_{\omega}$ and $\widetilde{H}$ is proportional to $\widetilde{N}_{\omega}=\tilde{a}_{\omega}^{\dagger} \tilde{a}_{\omega}$. The transformation generator that will be considered here is $\left[21,[22,25]^{3}\right.$.

$$
\mathbf{G}(\theta)=\sum_{i=1}^{3} \mathbf{g}_{i}(\theta)
$$

with,

$$
\begin{aligned}
& \mathbf{g}_{1}(\theta)=-\sum_{\omega=1} \theta_{1_{\omega}}\left(a_{\omega} \tilde{a}_{\omega}+\tilde{a}_{\omega}^{\dagger} a_{\omega}^{\dagger}\right) \\
& \mathbf{g}_{2}(\theta)=-\sum_{\omega=1} i \theta_{2_{\omega}}\left(a_{\omega} \tilde{a}_{\omega}-\tilde{a}_{\omega}^{\dagger} a_{\omega}^{\dagger}\right) \\
& \mathbf{g}_{3}(\theta)=-\sum_{\omega=1} \theta_{3_{\omega}}\left(a_{\omega}^{\dagger} a_{\omega}+\tilde{a}_{\omega} \tilde{a}^{\dagger}\right)
\end{aligned}
$$

Here $\theta_{j_{\omega}}, j=1,2,3$ denotes the set of transformation parameters. The generators written

\footnotetext{
${ }^{3}$ It must be noticed that the generator used here corresponds to the one that generates the inverse Bogoliubov transformation in [25] and [26], for example.
} 
in the last equations satisfy an $\mathrm{su}(1,1)$ algebra, and (29) can be rearranged as

$$
\mathbf{G}(\gamma)=-\sum_{\omega=1}\left[\gamma_{1_{\omega}} \tilde{a}_{\omega}^{\dagger} a_{\omega}^{\dagger}-\gamma_{2_{\omega}} a_{\omega} \cdot \tilde{a}_{\omega}+\gamma_{3_{\omega}}\left(a_{\omega}^{\dagger} a_{\omega}+\tilde{a}_{\omega} \tilde{a}_{\omega}^{\dagger}\right)\right]
$$

where the $\gamma$ 's coefficients are defined as

$$
\gamma_{1_{\omega}}=\theta_{1_{\omega}}-i \theta_{2_{\omega}}, \quad \gamma_{2_{\omega}}=-\gamma_{1_{\omega}}^{*}, \quad \gamma_{3_{\omega}}=\theta_{3_{\omega}}
$$

This generator carries out a unitary and canonical transformation, such that the creation and annihilation operators transform according to [26]

$$
\begin{aligned}
\left(\begin{array}{c}
a_{\omega}(\gamma) \\
\breve{a}_{\omega}^{\dagger}(\gamma)
\end{array}\right) & =e^{-i \mathbf{G}}\left(\begin{array}{c}
a_{\omega} \\
\tilde{a}_{\omega}^{\dagger}
\end{array}\right) e^{i \mathbf{G}}=\mathbb{B}_{\omega}\left(\begin{array}{c}
a_{\omega} \\
\tilde{a}_{\omega}^{\dagger}
\end{array}\right), \\
\left(a_{\omega}^{\dagger}(\gamma)-\breve{a}_{\omega}(\gamma)\right) & =\left(a_{\omega}^{\dagger}-\tilde{a}_{\omega}\right) \mathbb{B}_{\omega}^{-1},
\end{aligned}
$$

where the $S U(1,1)$ matrix transformation is given by

$$
\mathbb{B}_{\omega}=\left(\begin{array}{cc}
\mathfrak{u}_{\omega} & \mathfrak{v}_{\omega} \\
\mathfrak{v}_{\omega}^{*} & \mathfrak{u}_{\omega}^{*}
\end{array}\right), \quad\left|\mathfrak{u}_{\omega}\right|^{2}-\left|\mathfrak{v}_{\omega}\right|^{2}=1
$$

with elements [25]

$$
\mathfrak{u}_{\omega}=\cosh \left(i \Gamma_{\omega}\right)+\frac{\gamma_{3_{\omega}}}{\Gamma_{\omega}} \sinh \left(i \Gamma_{\omega}\right), \quad \mathfrak{v}_{\omega}=-\frac{\gamma_{1_{\omega}}}{\Gamma_{\omega}} \sinh \left(i \Gamma_{\omega}\right)
$$

and $\Gamma_{\omega}$ is defined by the following relation

$$
\Gamma_{\omega}^{2}=\gamma_{1_{\omega}} \gamma_{2_{\omega}}+\gamma_{3_{\omega}}^{2}
$$

A quite convenient way to write the Bogoliubov transformation matrix (36) arises if we make the polar decomposition $\mathfrak{u}_{\omega}=\left|\mathfrak{u}_{\omega}\right| e^{i \varphi_{\omega}}, \mathfrak{v}_{\omega}=\left|\mathfrak{v}_{\omega}\right| e^{i \phi_{\omega}}$, and rewrite the matrix elements in terms of the new parameters

$$
f_{\omega}=\frac{\left|\mathfrak{v}_{\omega}\right|^{2}}{\left|\mathfrak{u}_{\omega}\right|^{2}}, \quad \alpha_{\omega}=\frac{\log \left(\frac{\mathfrak{w}_{\omega}}{\mathfrak{u}_{\omega}}\right)}{\log \left(f_{\omega}\right)}=\frac{1}{2}+i \frac{\left(\phi_{\omega}-\varphi_{\omega}\right)}{\log \left(f_{\omega}\right)}, \quad s_{\omega}=i \varphi_{\omega}=\frac{1}{2} \log \left(\frac{\mathfrak{u}_{\omega}}{\mathfrak{u}_{\omega}^{*}}\right) .
$$

In fact, with these steps we can present the Bogoliubov matrix as [21, 24]

$$
\mathbb{B}_{n}=\frac{1}{\sqrt{1-f_{\omega}}}\left(\begin{array}{cc}
e^{s_{\omega}} & -f_{\omega}^{\alpha_{\omega}} e^{s_{\omega}} \\
-f_{\omega}^{\alpha_{\omega}^{*}} e^{-s_{\omega}} & e^{-s_{\omega}}
\end{array}\right)
$$

with $\alpha_{\omega}+\alpha_{\omega}^{*}=1$. 
As the Bogoliubov transformation is canonical, the gamma dependent operators obey the same commutation relations of (17). One can define a vacuum state for the transformed system as the state satisfying

$$
a_{\omega}(\gamma)|0(\gamma)\rangle=\widetilde{a}_{\omega}(\gamma)|0(\gamma)\rangle=0
$$

This expression, together with (39) and (40), gives rise to the following conditions:

$$
\begin{aligned}
e^{s_{\omega}}\left[a_{\omega}-f_{\omega}^{\alpha_{\omega}} \tilde{a}_{\omega}^{\dagger}\right]|0(\gamma)\rangle & =0, \\
e^{-s_{\omega}}\left[\tilde{a}_{\omega}-f_{\omega}^{\alpha_{\omega}^{*}} a_{\omega}^{\dagger}\right]|0(\gamma)\rangle & =0,
\end{aligned}
$$

At this moment, it is already possible to compare the conditions presented above with those defining the state $\left|B_{\text {hor }}\right\rangle$ in (26). However, let us explore a bit more the TFD formalism used here. The inverse of the Bogoliubov transformations (35) allow one to obtain that the number of modes of the string defined in the $\mathrm{R}$ quadrant is

$$
\bar{N}_{\omega}(\theta)=\left\langle 0(\beta)\left|a_{\omega} a_{\omega}^{\dagger}\right| 0(\theta)\right\rangle=\left|\mathfrak{v}_{\omega}\right|^{2}=\frac{\gamma_{1_{\omega}} \gamma_{2_{\omega}}}{\Gamma_{\omega}^{2}} \sinh ^{2}\left(i \Gamma_{\omega}\right)=\frac{f_{\omega}}{1-f_{\omega}}
$$

and similarly for the modes of type $\tilde{a}_{n}$ in the L quadrant. The transformation also entangles the states of the two independent Hilbert spaces [62, 63], and gives us a structure to the new vacuum, $|0(\gamma)\rangle$, as follows

$$
\left.\left.|0(\gamma)\rangle=e^{i \mathbf{G}}|0\rangle\right\rangle=\prod_{\omega=1}\left[e^{-s_{\omega}} \sqrt{1-f_{\omega}} e^{f_{\omega}^{\alpha_{\omega}} a_{\omega}^{\dagger} \tilde{a}_{\omega}^{\dagger}}\right]|0\rangle\right\rangle .
$$

For a suitable parameter choice, the state (45) is the string state defined in (27), and the relations (42), (43) are the relations (26), called thermal state conditions in the TFD formulation. Indeed, transformation parameters, as expected, encode information about the environment under which the system is subject (in our study, information about the model presented). Furthermore, TFD general approaches usually consider the $s_{\omega}$ and $\alpha_{\omega}$ parameters as being the same for all modes and deal with them as free parameters that can be fixed suitably for each situation. For the application considered here, it will be possible to verify that the $s_{\omega}$ parameter is no longer free, once it seems to contain information about the CFT boundary conditions. Note that $s_{\omega}$ is related to $\theta_{\omega}$, which defines the Neumann boundary conditions at cut off surface $\rho=\rho_{c}$. The $\alpha$ parameter is the only one that can be considered free in some sense. Rather, it is possible to consider it as being the same for all string modes $\left(\alpha_{\omega} \rightarrow \alpha\right)$ but also constrained by the relation $\alpha+\alpha^{*}=1$, that comes directly 
from the general TFD construction. From the thermo field point of view, the $\alpha$ constraint guarantees the KMS conditions as it can be verified directly following the proof for the nonunitary TFD formulation in [21], which might be useful in order to simplify the treatment of more engaged situations such as those where interactions or non-equilibrium effects are explicitly considered, as it is the case of the parameters' choice of the non-unitary TFD formulation 4 On the other hand, from the geometry's perspective, this constraint ensures the appropriate time periodicity, which is necessary to circumvent the conical singularity. Furthermore, $\alpha$ is related to the trace cyclicity in thermal statistical averages, as it seems to be the case here 5 However, as it will be shown, the expectation value of the system's observables at thermal equilibrium does not depend on $\alpha$. Finally, the $f_{\omega}$ parameter will contain information about the thermal distribution of the string modes, as it will be noticed.

Before specifying the parameters, let us introduce a very interesting operator which arises in this formalism. Consider the following gamma dependent operators defined on the $\mathrm{R}$ and L sectors, respectively

$$
\begin{aligned}
& K=-\sum_{\omega=1}\left[a_{\omega}^{\dagger} a_{\omega} \ln \left(\frac{\gamma_{1_{\omega}} \gamma_{2_{\omega}}}{\Gamma_{\omega}^{2}} \sinh ^{2}\left(i \Gamma_{\omega}\right)\right)-a_{\omega} a_{\omega}^{\dagger} \ln \left(1+\frac{\gamma_{1_{\omega}} \gamma_{2_{\omega}}}{\Gamma_{\omega}^{2}} \sinh ^{2}\left(i \Gamma_{\omega}\right)\right)\right], \\
& \widetilde{K}=-\sum_{\omega=1}\left[\tilde{a}_{\omega}^{\dagger} \tilde{a}_{\omega} \ln \left(\frac{\gamma_{1_{\omega}} \gamma_{2_{\omega}}}{\Gamma_{\omega}^{2}} \sinh ^{2}\left(i \Gamma_{\omega}\right)\right)-\tilde{a}_{\omega} \tilde{a}_{\omega}^{\dagger} \ln \left(1+\frac{\gamma_{1_{\omega}} \gamma_{2_{\omega}}}{\Gamma_{\omega}^{2}} \sinh ^{2}\left(i \Gamma_{\omega}\right)\right)\right],
\end{aligned}
$$

These operators are the entropy operators for the general unitary TFD formulation [28]. The extended operator $\widehat{K}=K-\widetilde{K}$ commutes with the Bogoliubov transformation generator (33).

The expectation value of the operator $K$ evaluated at the gamma dependent vacuum state can be calculated by usual methods and the result is given below:

$$
S(\gamma)=\langle 0(\theta)|K| 0(\theta)\rangle=\sum_{\omega=1}\left\{\left(1+\bar{N}_{\omega}\right) \ln \left(1+\bar{N}_{\omega}\right)-\bar{N}_{\omega} \ln \bar{N}_{\omega}\right\}
$$

where $\bar{N}_{\omega}$ was defined by (44); a similar expression is obtained for the L quadrant. The vacuum state (45) can be rewritten using these operators as followe 6

$$
\left.\left.|0(\gamma)\rangle=e^{\alpha K} e^{\sum_{\omega=1} a_{\omega}^{\dagger} \tilde{a}_{\omega}^{\dagger}}|0\rangle\right\rangle=e^{\alpha \widetilde{K}} e^{\sum_{\omega=1} a_{\omega}^{\dagger} \tilde{a}_{\omega}^{\dagger}}|0\rangle\right\rangle .
$$

\footnotetext{
${ }^{4}$ In fact, the non-unitary TFD formulation and that presented here share many formal features as it was pointed out in references [22] and 27] for instance.

${ }^{5}$ Notice that in expression (1), $\operatorname{Tr}[Q \rho]=\operatorname{Tr}\left[\rho^{(1-\alpha)} Q \rho^{(\alpha)}\right]$.

${ }^{6}$ Effectively, the state obtained using the $K$ operator differs from that arising from the use of the Bogoliubov generator by a phase, as it was pointed out in [28].
} 
or

$$
|0(\gamma)\rangle=\sum_{\omega=1} W_{\omega}^{\alpha}|\omega, \tilde{\omega}\rangle
$$

with

$$
W_{\omega}=\prod_{n=0} \frac{\left(\left|\mathfrak{v}_{\omega}\right|^{2}\right)^{n_{\omega}}}{\left(\left|\mathfrak{u}_{\omega}\right|^{2}\right)^{n_{\omega}+1}}=\left(1-f_{\omega}\right) \prod_{n=0}\left(f_{\omega}\right)^{n_{\omega}}, \quad \sum_{\omega=1} W_{\omega}=1,
$$

and (48) assumes the following form

$$
S(\gamma)=-\sum_{\omega=1} W_{\omega} \ln W_{\omega}
$$

As those results can be obtained by using $\tilde{K}$, we have the entropy operator defined in both quadrants. The whole vacuum $|0(\gamma)\rangle$ is an entanglement of states from both quadrants as it can be noticed explicitly in (50), and therefore expectation value of $K$ furnishes the entanglement entropy of the system.

The expressions presented earlier show that the presence of a horizon produces string entanglement entropy and the origin of the entanglement is the environment, in contrast with the usual quantum mechanical point of view, which attributes to the environment the loss of the entanglement. In this picture, the origin of macroscopic dissipation in the CFT side is related to the open string entanglement entropy caused by the AdS geometry.

Finally, the vacuum can be completely defined by minimizing the free energy

$$
\mathcal{F}=\mathcal{U}-\frac{1}{\beta} \mathcal{S}
$$

with respect to the transformation parameters [10], defining the open string thermal vacuum. Here $\mathcal{U}$ is given by the vacuum expectation value of the open string Hamiltonian in the thermal vacuum and $\mathcal{S}$ is the entropy given in (48). The solution for the Bogoliubov transformation parameters is given by the Bose-Einstein distribution. In fact, in this context $f_{\omega}=e^{-\beta \omega}$, and the expression (44) is now given by

$$
\bar{N}_{\omega}(\beta)=\frac{e^{-\beta \omega}}{1-e^{-\beta \omega}} .
$$

Taking into account these solutions and further relating the $s_{\omega}$ parameter in (39) with $\theta_{\omega}$ defined in (14), the state (45) is written as

$$
\left.|0(\beta)\rangle=\prod_{n=1} e^{-i \theta_{n}} \sqrt{1-e^{-\beta n}} \exp \left[\sum_{\omega=1} e^{-\alpha \omega \beta} a_{\omega}^{\dagger} \tilde{a}_{\omega}^{\dagger}\right]|0\rangle\right\rangle,
$$


(compare with that proposed in (27) as a solution of the gluing condition), and it is in fact the open string thermal vacuum for the system under consideration. Even knowing that the parameters $\theta_{\omega}$ are phases and $\alpha$ does not interfere in the thermal physics, the TFD general formulation shows itself quite fancy since it furnishes naturally a state with all the information of the model.

Also notice that once the Bogoliubov transformation parameter has been fixed as above, the expression (48) for the entropy takes the following form:

$$
\mathcal{S}(\beta)=\sum_{\omega=1} \beta \omega \bar{N}_{\omega}+\ln Z
$$

with

$$
Z=\prod_{\omega=1} \frac{1}{1-e^{-\beta \omega}} .
$$

Finite temperature supposedly violates conformal invariance; however, we expect that for any ordinary QFT, a conformal phase should exist for very high temperature. Following [64], for $\beta \ll 1$ we can consider [65, 66]

$$
\begin{gathered}
\sum_{\omega=1}^{\infty} \frac{\beta \omega}{e^{\beta \omega}-1} \rightarrow \int_{0}^{\infty} \frac{d x}{\beta} \frac{x}{e^{x}-1}=\frac{\pi^{2}}{6 \beta}, \\
\sum_{\omega=1}^{\infty} \ln \left(1-e^{-\beta \omega}\right) \rightarrow \int_{0}^{\infty} \frac{d x}{\beta} \ln \left(1-e^{-x}\right)=-\frac{\pi^{2}}{6 \beta},
\end{gathered}
$$

and (56) becomes

$$
S(\beta \ll 1)=\frac{\pi^{2}}{3 \beta}=2 \pi \sqrt{\frac{\bar{N}}{6}},
$$

where we have defined

$$
\bar{N}=\sum_{\omega} \omega \bar{N}_{\omega}=-\frac{\partial \ln Z}{\partial \beta},
$$

for $\bar{N}_{\omega}$ given in (54). The expression (60) is compatible with the Cardy formula for a central charge $c=1$. This reflects the fact that the conformal phase of the system is recovered in this limit and the value of the central charge corresponds to the single effective degree of freedom of a $D-1$-brane on which the string ends.

Once one has the general structure presented earlier, it is possible to obtain the free propagators for the model. Considering a fixed $\rho$ and following the TFD procedure, the 
propagators can be arranged as a matrix

$$
\mathbb{D}\left(t-t^{\prime}, \rho ; \gamma\right)=\left(\begin{array}{cc}
\left\langle 0(\gamma)\left|T\left[X(t, \rho) X\left(t^{\prime}, \rho\right)\right]\right| 0(\gamma)\right\rangle & \left\langle 0(\gamma)\left|T\left[X(t, \rho) \widetilde{X}\left(t^{\prime}, \rho\right)\right]\right| 0(\gamma)\right\rangle \\
\left\langle 0(\gamma)\left|T\left[\widetilde{X}(t, \rho) X\left(t^{\prime}, \rho\right)\right]\right| 0(\gamma)\right\rangle & \left\langle 0(\gamma)\left|T\left[\widetilde{X}(t, \rho) \widetilde{X}\left(t^{\prime}, \rho\right)\right]\right| 0(\gamma)\right\rangle
\end{array}\right),
$$

where $T$ stands for the time ordering of the worldsheet operator products in such a way that, as usual,

$$
T\left[X(t, \rho) X\left(t^{\prime}, \rho\right)\right]=\Theta\left(t-t^{\prime}\right) X(t, \rho) X\left(t^{\prime}, \rho\right)+\Theta\left(t^{\prime}-t\right) X\left(t^{\prime}, \rho\right) X(t, \rho)
$$

where $\Theta\left(t-t^{\prime}\right)$ is the Heaviside step function. As before, $X(t, \rho)$ and $\widetilde{X}(t, \rho)$ denote the solutions for the L and R quadrants, respectively. Using (15) and (21), the matrix entries are written in terms of the following propagators

$$
\begin{aligned}
D_{11}\left(t-t^{\prime}, \rho ; \gamma\right) & =\left\langle 0(\gamma)\left|X(t, \rho) X\left(t^{\prime}, \rho\right)\right| 0(\gamma)\right\rangle \\
& =\sum_{\omega>0} \frac{1}{1-f_{\omega}}\left\{u_{\omega}(t, \rho) u_{\omega}^{*}\left(t^{\prime}, \rho\right)+f_{\omega} u_{\omega}^{*}(t, \rho) u_{\omega}\left(t^{\prime}, \rho\right)\right\}, \\
D_{12}\left(t-t^{\prime}, \rho ; \gamma\right) & =\left\langle 0(\gamma)\left|X(t, \rho) \widetilde{X}\left(t^{\prime}, \rho\right)\right| 0(\gamma)\right\rangle \\
& =-\sum_{\omega>0} \frac{1}{1-f_{\omega}}\left\{e^{2 s_{\omega}} f_{\omega}^{\alpha} u_{\omega}(t, \rho) \tilde{u}_{\omega}\left(t^{\prime}, \rho\right)+e^{-2 s_{\omega}} f_{\omega}^{\alpha^{*}} u_{\omega}^{*}(t, \rho) \tilde{u}_{\omega}^{*}\left(t^{\prime}, \rho\right)\right\}, \\
D_{21}\left(t-t^{\prime}, \rho ; \gamma\right) & =\left\langle 0(\gamma)\left|\widetilde{X}(t, \rho) X\left(t^{\prime}, \rho\right)\right| 0(\gamma)\right\rangle \\
& =-\sum_{\omega>0} \frac{1}{1-f_{\omega}}\left\{e^{2 s_{\omega}} f_{\omega}^{\alpha} \tilde{u}_{\omega}(t, \rho) u_{\omega}\left(t^{\prime}, \rho\right)+e^{-2 s_{\omega}} f_{\omega}^{\alpha^{*}} \tilde{u}_{\omega}^{*}(t, \rho) u_{\omega}^{*}\left(t^{\prime}, \rho\right)\right\}, \\
D_{22}\left(t-t^{\prime}, \rho ; \gamma\right) & =\left\langle 0(\gamma)\left|\widetilde{X}(t, \rho) \widetilde{X}\left(t^{\prime}, \rho\right)\right| 0(\gamma)\right\rangle \\
& =\sum_{\omega>0} \frac{1}{1-f_{\omega}}\left\{\tilde{u}_{\omega}(t, \rho) \tilde{u}_{\omega}^{*}\left(t^{\prime}, \rho\right)+f_{\omega} \tilde{u}_{\omega}^{*}(t, \rho) \tilde{u}_{\omega}\left(t^{\prime}, \rho\right)\right\} .
\end{aligned}
$$

Considering the worldsheet defined in the boundary $\rho \rightarrow \rho_{c}$, the solutions (15) and (21) become

$$
\begin{array}{r}
X\left(t, \rho_{c}\right)=\sum_{\omega>0} \sqrt{\frac{2 \alpha^{\prime} \beta}{\ell^{2} \omega \log (1 / \epsilon)}}\left[\frac{1-i \nu}{1-i \rho_{c} \nu}\left(\frac{\rho_{c}-1}{\rho_{c}+1}\right)^{\frac{i \nu}{2}} e^{-i \omega t} a_{\omega}\right. \\
\left.+\frac{1+i \nu}{1+i \rho_{c} \nu}\left(\frac{\rho_{c}-1}{\rho_{c}+1}\right)^{\frac{-i \nu}{2}} e^{i \omega t} a_{\omega}^{\dagger}\right],
\end{array}
$$

for the $\mathrm{R}$ quadrant and

$$
\begin{array}{r}
\tilde{X}\left(t, \rho_{c}\right)=\sum_{\omega>0} \sqrt{\frac{2 \alpha^{\prime} \beta}{\ell^{2} \omega \log (1 / \epsilon)}}\left[\frac{1+i \nu}{1+i \rho_{c} \nu}\left(\frac{\rho_{c}-1}{\rho_{c}+1}\right)^{\frac{-i \nu}{2}} e^{i \omega t} \tilde{a}_{\omega}\right. \\
\left.+\frac{1-i \nu}{1-i \rho_{c} \nu}\left(\frac{\rho_{c}-1}{\rho_{c}+1}\right)^{\frac{i \nu}{2}} e^{-i \omega t} \tilde{a}_{\omega}^{\dagger}\right],
\end{array}
$$


for the $\mathrm{L}$ one. Therefore

$$
\begin{aligned}
& u_{\omega}\left(t, \rho_{c}\right)=\tilde{u}_{\omega}^{*}\left(t, \rho_{c}\right)=\frac{1-i \nu}{1-i \rho_{c}}\left(\frac{\rho_{c}-1}{\rho_{c}+1}\right)^{i \frac{\nu}{2}} e^{-i \omega t}, \\
& u_{\omega}^{*}\left(t, \rho_{c}\right)=\tilde{u}_{\omega}\left(t, \rho_{c}\right)=\frac{1+i \nu}{1+i \rho_{c}}\left(\frac{\rho_{c}-1}{\rho_{c}+1}\right)^{-i \frac{\nu}{2}} e^{i \omega t},
\end{aligned}
$$

Replacing the expressions written above in the propagators, we have

$$
\begin{aligned}
D_{11}\left(t-t^{\prime}, \rho_{c} ; \gamma\right)= & \frac{2 \alpha^{\prime} \beta}{\ell^{2} \log (1 / \epsilon)} \frac{1+\nu^{2}}{1+\rho_{c}^{2} \nu^{2}} \sum_{\omega>0} \frac{1}{\omega}\left\{e^{-i \omega\left(t-t^{\prime}\right)}+2 \frac{f_{\omega}}{1-f_{\omega}} \cos \left(\omega\left(t-t^{\prime}\right)\right)\right\} \\
D_{12}\left(t-t^{\prime}, \rho_{c} ; \gamma\right)= & -\frac{2 \alpha^{\prime} \beta}{\ell^{2} \log (1 / \epsilon)} \frac{1+\nu^{2}}{1+\rho_{c}^{2} \nu^{2}} \\
& \times \sum_{\omega>0} \frac{1}{\omega} \frac{1}{1-f_{\omega}}\left\{e^{2 s_{\omega}} f_{\omega}^{\alpha} e^{-i \omega\left(t-t^{\prime}\right)}+e^{-2 s_{\omega}} f_{\omega}^{\alpha^{*}} e^{i \omega\left(t-t^{\prime}\right)}\right\} \\
D_{21}\left(t-t^{\prime}, \rho_{c} ; \gamma\right)= & -\frac{2 \alpha^{\prime} \beta}{\ell^{2} \log (1 / \epsilon)} \frac{1+\nu^{2}}{1+\rho_{c}^{2} \nu^{2}} \\
& \times \sum_{\omega>0} \frac{1}{\omega} \frac{1}{1-f_{\omega}}\left\{e^{2 s_{\omega}} f_{\omega}^{\alpha} e^{i \omega\left(t-t^{\prime}\right)}+e^{-2 s_{\omega}} f_{\omega}^{\alpha^{*}} e^{-i \omega\left(t-t^{\prime}\right)}\right\} \\
D_{22}\left(t-t^{\prime} \rho_{c} ; \gamma\right)= & \frac{2 \alpha^{\prime} \beta}{\ell^{2} \log (1 / \epsilon)} \frac{1+\nu^{2}}{1+\rho_{c}^{2} \nu^{2}} \sum_{\omega>0} \frac{1}{\omega}\left\{e^{i \omega\left(t-t^{\prime}\right)}+2 \frac{f_{\omega}}{1-f_{\omega}} \cos \left(\omega\left(t-t^{\prime}\right)\right)\right\}
\end{aligned}
$$

The expression (72) is the propagator commonly used when thermal equilibrium is given. For example, making $f_{\omega}=e^{-\beta \omega}$ and $t^{\prime}=0$, we have (72) in a perfect match with the worldsheet thermal two point functions derived in [5] and considered in the calculation of the displacement of string endpoint, establishing the connection with the standard Brownian motion.

\section{CONCLUDING REMARKS}

In this work we have studied the string propagating in an AdS Schwarzschild spacetime from the point of view of a thermal theory in the worldsheet. In particular, the approach to the Brownian motion of a quark in a CFT fluid at finite temperature developed in Ref. [5] was reinforced in this work since the main computations were reproduced. The construction presented here is based on Israel-Maldacena's picture, where the AdS-Schwarzschild spacetime is maximally extended along with the fundamental string solution: we have extended it through natural gluing conditions imposed on an effective surface near the event horizon. We have pointed out that the gluing conditions of the string at the horizon define a boundary state, which is exactly the thermal vacuum of the Thermo Field Dynamics 
(TFD), and the connection of this point of view with unitary SU(1,1) TFD formulation was emphasized here. It was shown that all information about the bulk and the board are present in the $\mathrm{SU}(1,1)$ parameters. As the string boundary state is an entangled state, we calculated the entanglement entropy, which in this case coincides with the thermodynamic entropy. We show that in, the high temperature limit, the result agrees with the Cardy formula, reflecting the fact that the conformal phase is recovered at high temperatures. Also, the canonical approach of TFD was explored to calculate the worldsheet real time thermal Green's functions. An important aspect of the framework explored here is the presence of a string boundary state. This kind of state can be used to study the precise microscopic structure of the stretched horizon. In fact, as noticed in Ref. [59], this state approaches the following pure but coherent one:

$$
\left.|0(\gamma)\rangle \sim e^{\sum_{\omega=1} a_{\omega}^{\dagger} \tilde{a}_{\omega}^{\dagger}}|0\rangle\right\rangle
$$

in the limit $K \rightarrow 0$ (or $\alpha \rightarrow \infty)$ of (49), so it describes a macroscopic (semiclassical) object. Because the open string ends on this surface, we can go to the closed string channel and this state can clearly be identified with the state of a $D_{p}$-brane $(p=D-1)$ [61], which reinforces the idea that the stretched horizon may be described as a real $D_{p}$-brane. A similar scenario was studied in Ref. [56] in the context of pp-wave time-dependent background, where it was shown that, for asymptotically flat observers, the closed string vacuum close to the singularity appears as a boundary state, which is in fact a D-brane described in the closed string channel.

In a forthcoming work we shall try to describe the Brownian motion and the stochastic effects (ruled out by a Langevin equation) in terms of microscopic aspects of the string gluing, and by considering interaction between both strings in the contact (horizon) surface. In fact, the velocity of the string endpoint is high if it does not fall into the black hole, so more than the quadratic order should be taken into account in the Nambu-Goto action as the parameter $\epsilon(\equiv \rho-1)$ approaches to zero. A sketch to argument dissipative effects as $\epsilon \rightarrow 0$ in this framework is indeed the following: non-gaussian terms in the action are not invariant under Bogoliubov transformations in general; so for generic frames (accelerated with respect to the horizon), products of tilde with non-tilde fields are induced by the Bogoliubov transformation of these terms, which typically describes dissipation in TFD, [8, 58, 67 69]. A similar idea was applied to construct a string vertex state for a Rindler 
horizon [8].

We conclude this work by pointing out that some more refined interpretations arise from the present construction. For instance, it suggests that the dual picture of the AdS stretched horizon might be described as an entangled state of two (decoupled) heavy quarks in the hydrodynamic regime of CFT's fluids.

\section{ACKNOWLEDGMENTS}

Daniel Luiz Nedel would like to thank CNPq, grant 501317/2009-0, for financial support. MBC was partially supported by: CONICET PIP 2010-0396 and ANPCyT PICT 2007-0849.

[1] J. M. Maldacena, "The large N limit of superconformal field theories and supergravity", Adv. Theor. Math. Phys. 2 (1998) 231 [Int. J. Theor. Phys. 38 (1999) 1113] [arXiv:hepth/9711200].

[2] S. S. Gubser, I. R. Klebanov and A. M. Polyakov, "Gauge theory correlators from noncritical string theory", Phys. Lett. B 428, 105 (1998) [arXiv:hep-th/9802109].

[3] E.Witten, "Anti-de Sitter space and holography", Adv. Theor. Math. Phys. 2, 253 (1998) [arXiv:hep-th/9802150].

[4] O. Aharony, S. S. Gubser, J. M. Maldacena, H. Ooguri and Y. Oz, "Large N field theories, string theory and gravity", Phys. Rept. 323 (2000) 183 [arXiv:hep-th/9905111].

[5] J. de Boer, V. E. Hubeny, M. Rangamani and M. Shigemori, "Brownian motion in AdS/CFT," JHEP 0907 (2009) 094 [arXiv:0812.5112 [hep-th]].

[6] D. T. Son and D. Teaney, "Thermal Noise and Stochastic Strings in AdS/CFT", JHEP 0907 (2009) 021 [arXiv:0901.2338 [hep-th]].

[7] G. C. Giecold, E. Iancu and A. H. Mueller, "Stochastic trailing string and Langevin dynamics from AdS/CFT," JHEP 0907, 033 (2009) [arXiv:0903.1840 [hep-th]].

[8] M. C. Batoni Abdalla, M. Botta Cantcheff and D. L. Nedel, "Strings in horizons, dissipation and a simple interpretation of the Hagedorn temperature," Eur. Phys. J. C 54 (2008) 311 [hep-th/0703064 [HEP-TH]].

[9] C. P. Herzog and D. T. Son, "Schwinger-Keldysh propagators from AdS/CFT correspondence", JHEP 0303 (2003) 046 [hep-th/0212072]. 
[10] Y. Takahasi and H. Umezawa, "Thermo Field Dynamics", Collect. Phenom. 2 (1975) 55.

[11] W. Israel, "Thermo field dynamics of black holes," Phys. Lett. A 57 (1976) 107.

[12] J. M. Maldacena, "Eternal black holes in anti-de Sitter," JHEP 0304 (2003) 021 [hepth/0106112].

[13] R. Haag, "Local Quantum Physics: Fields, Particle, Algebra" (Spring-Verlag, New Yourk, 1992).

[14] G. G. Emch, "Algebraic Methods in Statistical and Quantum Field Theory" (Jonh Wiley, New York, 1972)

[15] I. Ojima, "Gauge Fields at Finite Temperatures: Thermo Field Dynamics, KMS Condition and their Extension to Gauge Theories," Annals Phys. 137 (1981) 1.

[16] N. P. Landsman and C. G. van Weert, "Real and Imaginary Time Field Theory at Finite Temperature and Density," Phys. Rept. 145 (1987) 141.

[17] A. E. Santana and F. C. Khanna, "Lie groups and thermal field theory," Phys. Lett. A 203 (1995) 68.

[18] A. E. Santana, F. C. Khanna, H. Chu and Y. C. Chang, "Thermal lie groups, classical mechanics, and thermofield dynamics," Annals Phys. 249 (1996) 481.

[19] M. C. B. Abdalla, A. L. Gadelha and D. L. Nedel, "Closed string thermal torus from thermofield dynamics," Phys. Lett. B 613 (2005) 213 [hep-th/0410068].

[20] F. C. Khanna, A. P. C. Malbouisson, J. M. C. Malbouisson and A. R. Santana, "Thermal quantum field theory - Algebraic aspects and applications," (Hackensack-NJ, World Scientific, 2009).

[21] H. Umezawa, "Advanced field theory: Micro, macro, and thermal physics", New York, USA: AIP (1993) $238 \mathrm{p}$

[22] H. Chu and H. Umezawa, "A Unified formalism of thermal quantum field theory", Int. J. Mod. Phys. A 9 (1994) 2363.

[23] P. Elmfors and H. Umezawa, "Generalizations of the thermal Bogolyubov transformation", Physica A 202 (1994) 577. [hep-th/9304089].

[24] P. A. Henning, "Thermo field dynamics for quantum fields with continuous mass spectrum", Phys. Rept. 253 (1995) 235.

[25] M. C. B. Abdalla, A. L. Gadelha and I. V. Vancea, "On the SU(1,1) thermal group of bosonic strings and D-branes", Phys. Rev. D 66 (2002) 065005. [arXiv:hep-th/0203222]. 
[26] M. C. B. Abdalla and A. L. Gadelha, "General unitary SU(1,1) TFD formulation," Phys. Lett. A 322 (2004) 31 [hep-th/0309254].

[27] M. C. B. Abdalla, A. L. Gadelha and I. V. Vancea, "Bosonic Dp-branes at finite temperature in TFD approach," Nucl. Phys. Proc. Suppl. 127 (2004) 92.

[28] M. C. B. Abdalla, A. L. Gadelha and D. L. Nedel, "On the entropy operator for the general SU(1,1) TFD formulation", Phys. Lett. A 334 (2005) 123 [hep-th/0409116].

[29] M. C. B. Abdalla, A. L. Gadelha and D. L. Nedel, "General unitary TFD formulation for superstrings," PoS WC 2004 (2004) 032 [hep-th/0412128].

[30] Y. Leblanc, "String Field Theory At Finite Temperature," Phys. Rev. D 36, 1780 (1987).

[31] Y. Leblanc, "Finite Temperature Amplitudes In Open String Systems," Phys. Rev. D 371547 (1988).

[32] Y. Leblanc, "Cosmological Aspects Of The Heterotic String Above The Hagedorn Temperature," Phys. Rev. D 38, 3087 (1988).

[33] Y. Leblanc, "Improved Integral Representation For The Finite-Temperature Propagator In String Theory," Phys. Rev. D 391139 (1989).

[34] H. Fujisaki, K. Nakagawa and I. Shirai, "Comments On The Thermal Neveu-Schwarz-Ramond Superstring," Prog. Theor. Phys. 81, 570 (1989).

[35] H. Fujisaki and K. Nakagawa, "The Thermal Stability Of Renormalization Of Open Bosonic Strings," Prog. Theor. Phys. 82, 236 (1989).

[36] H. Fujisaki and K. Nakagawa, "Renormalizability Of Open Bosonic Thermal Strings," Prog. Theor. Phys. 82, 1017 (1989).

[37] Y. Leblanc, M. Knecht and J. C. Wallet, "Regularization Of Finite Temperature String Theories," Phys. Lett. B 237357 (1990).

[38] Y. Leblanc, "Generalized Mcclain-Roth-O'brien-Tan Theorem And The String Free Energy At Higher Genus," Phys. Rev. Lett. 64831 (1990).

[39] H. Fujisaki and K. Nakagawa, "Infrared Behavior Of The Type I Neveu-Schwarz-Ramond Open Thermal Superstring," Europhys. Lett. 14, 737 (1991).

[40] H. Fujisaki and K. Nakagawa, "Infrared behavior of the type II closed thermal superstring," Europhys. Lett. 20, 677 (1992).

[41] H. Fujisaki, "Dimensional regularization of the closed bosonic thermal string," Europhys. Lett. 28, 623 (1994). 
[42] H. Fujisaki, "Thermofield dynamics of the heterotic string: Physical aspects of the thermal duality," Europhys. Lett. 39, 479 (1997). [arXiv:hep-th/9704180].

[43] M. C. B. Abdalla, A. L. Gadelha and I. V. Vancea, "On (b,c)-system at finite temperature in thermo field approach," Phys. Lett. A 273, 235 (2000). [arXiv:hep-th/0003209].

[44] I. V. Vancea, "Bosonic D-branes at finite temperature," Phys. Lett. B 487, 175 (2000). [arXiv:hep-th/0006228].

[45] M. C. B. Abdalla, A. L. Gadelha and I. V. Vancea, "Bosonic D-branes at finite temperature with an external field," Phys. Rev. D 64, 086005 (2001). [arXiv:hep-th/0104068].

[46] M. C. B. Abdalla, A. L. Gadelha and I. V. Vancea, "TFD approach for bosonic strings and D(p)-branes," Int. J. Mod. Phys. A 18, 2109 (2003). [arXiv:hep-th/0301249].

[47] M. C. B. Abdalla, A. L. Gadelha and I. V. Vancea, "D-branes at finite temperature in TFD," arXiv:hep-th/0308114.

[48] D. L. Nedel, M. C. B. Abdalla and A. L. Gadelha, "Superstring in a pp-wave background at finite temperature: TFD approach," Phys. Lett. B 598, 121 (2004). [arXiv:hep-th/0405258].

[49] M. C. B. Abdalla, A. L. Gadelha and D. L. Nedel, "Perspectives of TFD on string theory," PoS WC2004, 020 (2004). [arXiv:hep-th/0412134].

[50] M. C. B. Abdalla, A. L. Gadelha and D. L. Nedel, "PP-wave light-cone free string field theory at finite temperature," JHEP 0510, 063 (2005). [arXiv:hep-th/0508195].

[51] E. L. Graca and I. V. Vancea, "Thermal string vacuum in black-hole AdS spacetime," hepth/0505210.

[52] A. L. Gadelha, D. Z. Marchioro and D. L. Nedel, "Entanglement and entropy operator for strings in pp-wave time dependent background," Phys. Lett. B 639 (2006) 383 [hepth/0605237].

[53] I. V. Vancea, "Thermal D-brane boundary states from Green-Schwarz superstrings," Phys. Rev. D 74, 086002 (2006). [arXiv:hep-th/0607167].

[54] H. Belich, E. L. Graca, M. A. Santos and I. V. Vancea, "Semiclassical thermal string in the black-hole AdS spacetime," JHEP 0702, 037 (2007). [arXiv:hep-th/0610271].

[55] I. V. Vancea, "Thermal $D$-brane boundary states from type IIB Green-Schwarz superstring in $p p$-wave background," Int. J. Mod. Phys. A 23, 4485 (2008). [arXiv:0712.1569 [hep-th]].

[56] D. Z. Marchioro and D. L. Nedel, "Observer dependent D-brane for strings propagating in pp-wave time dependent background," Eur. Phys. J. C 55 (2008) 343 [arXiv:0711.0556 [hep- 
th]].

[57] R. Nardi, M. A. Santos and I. V. Vancea, "Thermal magnetized D-branes on $R^{1, p} \times T^{d-p-1}$ in the generalized Thermo Field Dynamics approach," J. Phys. A 44, 235403 (2011) [arXiv:1011.0574 [hep-th]].

[58] R. Nardi and I. V. Vancea, "Nonequilibrium dynamics of strings in time-dependent plane wave backgrounds," Nucl. Phys. B 859 (2012) 269 [arXiv:1112.4389 [hep-th]].

[59] M. Botta Cantcheff, "String Entanglement and D-branes as Pure States", Phys. Rev. D 80 (2009) 046001, [hep-th/0906.3049].

[60] Marcelo Botta Cantcheff "Emergent spacetime, and a model for unitary gravitational collapse in AdS" arXiv:1110.0867v1 [hep-th]; see also "Quantum states of the spacetime, and formation of black holes in AdS" arXiv:1205.3113 [hep-th], version presented in the Gravity Research Competition 2012.

[61] M Botta Cantcheff, "D-branes as coherent states in the open string channel", Eur. Phys. J. C 55 (2008) 517- 523 [arXiv:0710.3186 [hep-th]].

[62] A. Iorio, G. Lambiase and G. Vitiello, "Entangled quantum fields near the event horizon and entropy", Annals Phys. 309 (2004) 151.

[63] A. E. Santana, F. C. Khanna and M. Revzen, "Entropy of entangled states and SU(1,1) and SU(2) symmetries", Phys. Rev. A 65 (2002) 032119.

[64] Y. Sasai and A. Zahabi, "Shear viscosity of a highly excited string and the black hole membrane paradigm", Phys. Rev. D 83 (2011) 026002 [arXiv:1010.5380 [hep-th]].

[65] T. Damour and G. Veneziano, "Selfgravitating fundamental strings and black holes", Nucl. Phys. B 568 (2000) 93 [hep-th/9907030].

[66] I. S. Gradshtein and I. M. Ryzhik, "Tables of series, products, and integrals" (Berlin, Deutscher Verlag der Wissenschaften, 1963).

[67] T. Arimitsu and H. Umezawa, "A General Formulation Of Nonequilibrium Thermo Field Dynamics," Prog. Theor. Phys. 74, 429 (1985).

[68] T. Arimitsu and H. Umezawa, "Nonequilibrium Thermo Field Dynamics", Prog. Theor. Phys. 77, 32 (1987).

[69] Y. Mizutani, T. Inagaki, Y. Nakamura and Y. Yamanaka, "Canonical Quantization for a Relativistic Neutral Scalar Field in Non-equilibrium Thermo Field Dynamics, Prog. Theor. Phys. 126, 681 (2012) [arXiv:1105.5952 [hep-th]]. 